\title{
On $\rho$-Constrained Upward Topological Book Embeddings
}

\author{
Tamara Mchedlidze and Antonios Symvonis \\ Dept. of Mathematics, National Technical University of Athens, Athens, Greece \\ \{mchet, symvonis\}@math.ntua.gr
}

\section{Introduction}

Giordano, Liotta and Whitesides [1] developed an algorithm that, given an embedded planar st-digraph and a topological numbering $\rho$ of its vertices, computes in $O\left(n^{2}\right)$ time a $\rho$-constrained upward topological book embedding with at most $2 n-4$ spine crossings per edge. The number of spine crossings per edge is asymptotically worst case optimal.

In this poster, we present improved results with respect to the number of spine crossings per edge and the time required to compute the book embedding. Firstly, for any embedded planar st-digraph $G$ and any topological numbering $\rho$ of its vertices, there exists a $\rho$-constrained upward topological book embedding with at most $n-3$ spine crossings per edge and, moreover, $n-3$ spine crossing per edge are required for some graphs. In this result, we allow edge $(s, t)$ to be internal in the embedding of the graph. If edge $(s, t)$ is always on the external face, the corresponding number of spine crossings reduces to at most $n-4$ and is worst case optimal. Secondly, a $\rho$-contrained upward topological book embedding with minimum number of spine crossings and at most $n-3$ spine crossings per edge can be computed by an output sensitive algorithm in $O(\alpha+n)$ time, where $\alpha$ is the total number of spine crossings.

\section{Results}

The problem of Acyclic HP-completion with crossing minimization problem (for short, Acyclic-HPCCM) was defined in [2] as follows: Given an embedded upward planar st-digraph $G=(V, E)$ and a non-negative integer $c$, the AcyclicHPCCM problem asks whether there exists a Hamiltonian Path Completion set (for short, $H P$-completion set) $E_{c}$ and a drawing $\Gamma\left(G^{\prime}\right)$ of graph $G^{\prime}=\left(V, E \cup E_{c}\right)$ such that (i) $G^{\prime}$ remains acyclic and has a hamiltonian path from vertex $s$ to vertex $t$, (ii) $\Gamma\left(G^{\prime}\right)$ preserves the drawing of the embedded planar graph $G$ and, (iii) $\Gamma\left(G^{\prime}\right)$ has at most $c$ edge crossings. In Theorem 2 of [2, the equivalence between the Acyclic-HPCCM problem and the problem of determining an upward topological book embedding with minimum number of spine crossings was established. Moreover, in the proof of the theorem it was shown that if we are given an acyclic HP-completion set $E_{c}$ and an embedding of its edges on the original drawing of $G$ causing $c$ edge crossings, then there is an upward topological 
book embedding of $G$ with $c$ spine crossings and vice versa. In the construction supporting the proof, the crossings of the original edges of $G$ with the edges of the HP-completion set are exactly the crossings of the original edges with the spine in the constructed upward topological book embedding. In the following theorems, we assume that the edges of $E_{c}$ do not cross each other and that each pair of edges in the HP-completed drawing cross at most once.

Theorem 1. Let $G$ be an embedded planar st-digraph and $E_{c}$ be an acyclic $H P$-completion set of $G$. Then, there exists a unique upward drawing $\Gamma\left(G^{\prime}\right)$ of $G^{\prime}=\left(V, E \cup E_{c}\right)$ that respects the original embedding. If edge $(s, t)$ is not on the external face of $G, \Gamma\left(G^{\prime}\right)$ has at most $n-3$ crossings per edge, otherwise it has at most $n-4$ crossings per edge. Moreover, there exist embedded graphs and acyclic HP-completions sets that require so many edge crossings.

Theorem 2. Let $G$ be an embedded planar st-digraph, $E_{c}$ be an acyclic $H P$ completion set of $G$ and $P$ be the implied Hamiltonian path. Then, the unique upward drawing $\Gamma\left(G^{\prime}\right)$ of $G^{\prime}=\left(V, E \cup E_{c}\right)$ that respects the original embedding can be computed in $O(n+\alpha)$ time, where $\alpha$ is the total number of edge crossings.

Let $\rho=\left(s=v_{1}, v_{2}, \ldots, v_{n}=t\right)$ be a topological ordering of the vertices of $G$. Then, observe that the edge set $E^{\rho}=\left\{\left(v_{i}, v_{i+1}\right) \mid\left(v_{i}, v_{i+1}\right) \notin E, 1 \leq i<n\right\}$ is an HP-completion set for $G$. Based on this fact, Theorems 1 and 2 and the equivalence between the Acyclic-HPCCM and the book embedding problems established in [2], we can state the following theorems:

Theorem 3. Let $G$ be an embedded planar st-digraph and $\rho$ be a topological numbering of $G$. Then, $G$ admits a unique $\rho$-constrained upward topological book embedding with at most $n-3$ spine crossings per edge for the case where edge $(s, t)$ is not on the external face, otherwise it admits a embedding with at most $n-4$ spine crossings per edge. Moreover, there exist embedded graphs and topological orderings that require so many spine crossings.

Theorem 4. Let $G$ be an embedded planar st-digraph and $\rho$ be a topological numbering of $G$. Then, the unique $\rho$-constrained upward topological book embedding of $G$ (with a minimum number of spine crossings) can be computed in $O(n+\alpha)$ time, where $\alpha$ is the total number of spine crossings.

We note that the improved results on $\rho$-constrained upward topological book embeddings can be used to also improve results presented in [1] regarding upward point set embeddability.

\section{References}

1. Giordano, F., Liotta, G., Whitesides, S.H.: Embeddability Problems for Upward Planar Digraphs. In: Tollis, I.G., Patrignani, M. (eds.) GD 2008. LNCS, vol. 5417, pp. 242-253. Springer, Heidelberg (2009)

2. Mchedlidze, T., Symvonis, A.: Crossing-optimal acyclic hamiltonian path completion and its application to upward topological book embeddings. In: Das, S., Uehara, R. (eds.) WALCOM 2009. LNCS, vol. 5431, pp. 250-261. Springer, Heidelberg (2009) 